\title{
Are traditional NSAIDs prescribed appropriately among French elderly with osteoarthritis? Results from the CADEUS cohort
}

Sinem Ezgi Gulmez, Cécile Droz-Perroteau, Régis Lassalle, Patrick Blin, Bernard Bégaud, Michel Rossignol, Nicholas Moore, Annie Fourrier-Réglat*; On behalf of the CADEUS study group

Sinem Ezgi Gulmez, Associate Professor of Pharmacology

INSERM CIC 0005 Pharmaco-Epidémiologie, Service de Pharmacologie, Université de Bordeaux - CHU de Bordeaux,

Bâtiment Le Tondu - Case 41, 146, Rue Léo Saignat, 33076 Bordeaux Cedex, France

Cécile Droz-Perroteau, Pharmacoepidemiology Unit Chief Operating Officer Service de Pharmacologie, Université de Bordeaux, INSERM CIC 0005, INSERM Unité U657, IFR 99,

Bâtiment Le Tondu - Case 41, 146, Rue Léo Saignat, 33076 Bordeaux Cedex, France

Régis Lassalle, Principal Statistician and Database Manager

INSERM CIC 0005 Pharmaco-Epidémiologie, Service de Pharmacologie, Université de Bordeaux - CHU de Bordeaux,

Bâtiment Le Tondu - Case 41, 146, Rue Léo Saignat, 33076 Bordeaux Cedex, France 
Patrick Blin, Consultant Pharmacoepidemiologist

INSERM CIC 0005 Pharmaco-Epidémiologie, Service de Pharmacologie, Université de Bordeaux - CHU de Bordeaux,

Bâtiment Le Tondu - Case 41, 146, Rue Léo Saignat, 33076 Bordeaux Cedex, France

Bernard Bégaud, Professor of Pharmacology

Service de Pharmacologie, Université de Bordeaux, INSERM CIC 0005, INSERM Unité U657, IFR 99, CHU de Bordeaux,

Bâtiment 1A Zone nord Site Carreire, Case 36

33076 Bordeaux, France

Michel Rossignol, Associate Professor of Epidemiology

Department of Epidemiology, Biostatistics and Occupational Health, McGill University, 4820 Boul. Saint-Laurent

Montreal, Quebec Canada.

Nicholas Moore, Professor of Pharmacology $y^{1,2,3,4}$

Service de Pharmacologie, Université de Bordeaux, INSERM CIC 0005, INSERM Unité U657, IFR 99, CHU de Bordeaux,

Bâtiment 1A Zone nord Site Carreire, Case 36

33076 Bordeaux, France

Annie Fourrier-Réglat, Pharmacoepidemiology Unit Head

Service de Pharmacologie, Université de Bordeaux, INSERM CIC 0005, INSERM Unité 
U657, IFR 99, CHU de Bordeaux,

Bâtiment 1A Zone nord Site Carreire, Case 36

33076 Bordeaux, France

*Correspondence:

Annie Fourrier-Reglat

Service Hospitalo-Universitaire de Pharmacologie, Université de Bordeaux

Zone Nord Carreire - Batîment 1A - Case 36

146, rue Léo Saignat

33076 Bordeaux Cedex, FRANCE

Tel: +33 (0) 557574656

Fax: +33 (0) 557574671

E-mail: annie.fourrier@pharmaco.u-bordeaux2.fr 


\section{Abstract}

Aim: To describe inappropriate use of traditional non-steroidal anti-inflammatory drugs (tNSAIDs) in elderly subjects in the CADEUS cohort using Beers' 2003 criteria modified by recommendations from the French medicines agency.

Methods: Within the CADEUS cohort (23,217 subjects), 1,851 were $\geq 65$ years old with a diagnosis of osteoarthritis (OA) and a dispensation of a tNSAID at least once in the 6 months before index date. Data were obtained from the French national reimbursement database, patient and prescriber questionnaires. Beers criteria for inappropriate use were modified to include all tNSAIDs and long-term high-dose use was defined as having at least 5 tNSAID dispensations over 6 months with a gap of less than 45 days between each of these, and when a gap was greater than 45 days, medicine availability $>50 \%$ (i.e. DDD delivered/theoretical DDD for the gap.

Results: The most frequently dispensed tNSAIDs were piroxicam (25\%), diclofenac (24\%), ibuprofen (18\%), ketoprofen (18\%), and naproxen (10\%). Of the study population $1.5 \%$ were dispensed indomethacin; $15 \%$ two tNSAIDs; $15 \%$ a tNSAIDs with a platelet aggregation inhibitor; $4.6 \%$ a tNSAID with low-dose aspirin, and $0.2 \%$ with VKA. Eighteen percent of the study population were high-dose and long-term users of tNSAIDs, and $70 \%$ of these were dispensed a proton pump inhibitor.

Conclusions: The most common inappropriate tNSAIDs dispensation was the co-prescription of two different tNSAIDs within a month, or of a platelet aggregation inhibitor. The real-life consequences of these needs to be ascertained and it would be interesting to update Beers criteria.

Key Words: CADEUS, non-steroidal anti-inflammatory drugs, Beers criteria, elderly, inappropriate prescribing. 


\section{Introduction}

Non-steroidal anti-inflammatory drugs (NSAIDs) are among the most commonly used drugs,

to relieve pain and inflammation, with three main therapeutic areas: inflammatory rheumatisms (rheumatoid arthritis (RA), ankylosing spondylarthritis or psoriatic arthritis), osteoarthritis (OA), and common painful disorders such as headache, minor trauma or tendinitis, dysmenorrhoea. The patterns of tNSAID use are quite different between the three main indication domains, with essentially continuous use in rheumatic diseases; intermittent chronic use (100+ defined daily dose [DDD] per year) in OA, and a few days at usually lower doses in common pain. The age groups concerned are also quite different, OA patients being usually older, with a mean age of 66 years compared to 55 years for RA, 43 years for common pain. Two-thirds of elderly patients ( $\geq 65$ years of age) taking tNSAIDs do so for OA (approximately 35\%) or back pain (approximately 32\%), [1-3].

NSAIDs inhibit cyclo-oxygenase enzymes, resulting in an inhibition of the synthesis of prostaglandins [4]. This in turn increases the susceptibility of the stomach mucosa to acid aggression, but also may result in renal dysfunction with an in increased plasma volume. More recently, following the marketing of the COX-2 specific inhibitors [5, 6], an increased risk of myocardial infarction has been suggested for all NSAIDs [7].

Age above 65 years, previous gastrointestinal (GI) history (oesophageal ulcer, gastroduodenal ulcer, perforated ulcer, or upper gastrointestinal bleeding), prolonged use and high doses of NSAIDs are among the well-known risk factors for GI bleeding. In addition, the risk is higher with certain drugs with long half-lives such as piroxicam, or a strong COX-1 inhibition such as with naproxen or indomethacin. GI bleeding in the elderly is a matter of concern because of the increased frailty of these patients, of the commonly concomitant vascular diseases, and the increased risk of acute renal failure related to bleeding-induced hypovolemia magnified by renal COX-2 inhibition. Renal failure as well as aggravated (or 
even induced) heart failure are also the potential and serious side effects of NSAIDs. Accordingly, strong recommendations have been made to ensure proper prescription of NSAIDs in patients who need them, embodied in the Beers criteria (modified in 2002) [8-10], in regulatory recommendations $[11,12]$, and in the indications for gastroprotection with proton pump inhibitors in certain countries.

The updated 2003 Beers criteria define the inappropriate medication use in older adults either independently or considering diagnosis or conditions [10]. According to the NSAIDs listed in Beers criteria and its rationale, all use of indomethacin and ketorolac should be avoided independent of diagnoses or conditions. Additionally, long-term use of high-dose tNSAIDs (naproxen, oxaprozin, piroxicam) should be avoided as they have potential for renal failure, GI bleeding, hypertension, and heart failure. Patients with gastric or duodenal ulcers should avoid using tNSAID (coxibs excluded) and aspirin $>325 \mathrm{mg} /$ day since they may exacerbate existing ulcer disease or create new ulcers. Furthermore, patients with blood clotting disorders (including anticoagulant therapy) should use neither aspirin nor NSAIDs (tNSAIDs or coxibs) as well as dipyridamole, ticlopidine, clopidogrel since there is an increased risk of bleeding through multiple mechanisms of action [10].

The French Medicines Agency (Agence française de sécurité sanitaire des produits de santé, Afssaps), has published a recommendation for the rational prescribing of NSAIDs and paid attention to the prescription of ketoprofen and piroxicam [11]. Ketorolac is no longer on the French Market. Afssaps recommends the use of NSAIDs with the minimum effective dose and for the shortest possible duration of time. Contraindications can be summarised as follows: NSAIDs (tNSAIDs or coxibs) should not be prescribed if the patient has a history of peptic ulcer disease, NSAID-induced GI bleeding or severe heart failure. To avoid drug-drug interactions, NSAIDs (tNSAID, coxibs or aspirin at anti-inflammatory doses $\geq 325 \mathrm{mg} /$ day) 
should not be co-prescribed. It is also recommended to take into account that co-prescription of NSAIDs with platelet aggregation inhibitors increases the risk of GI bleeding.

In order to study the use of COX-2 selective NSAIDs celecoxib and rofecoxib compared to that of traditional non-selective NSAIDs (tNSAID), the French regulatory authorities commissioned a large-scale study, CADEUS (COX-2 inhibitors and NSAIDs: description of users), whereby patients were identified by the dispensation of a coxib or tNSAID; further information was obtained from the prescriber of the patient. Dispensation of other drugs and use of healthcare resources during the 6 months before and after the index selection date were obtained from the national reimbursement database. This cohort has already been described [2], as well as the determinants of coxib use, showing that they were used almost exclusively in the patients with rheumatoid arthritis. The main drivers of coxib prescription were age, female gender, previous gastrointestinal history, and previous use of proton pump inhibitors (PPI) [3].

In the CADEUS cohort, the main indication of NSAID prescription among the patients aged $>65$ years was OA. We study here the elderly patients using tNSAIDs for OA in the CADEUS study to test the adherence to Beer's criteria and to the recommendations of Afssaps. 


\section{Materials and methods}

\section{Data sources: CADEUS study description}

CADEUS is a national prospective cohort study of the characteristics of random sample of patients registered in the database of the French National Healthcare Insurance System for salaried persons (CNAM-TS), which covers $80 \%$ of the French population, and who submitted a claim for celecoxib, rofecoxib or traditional non-selective NSAIDs (tNSAIDs) during the study period 01/08/2003 - 31/07/2004. Patients were assigned an index date equivalent to the date of celecoxib, rofecoxib or tNSAID dispensation. Study methods have been described in detail elsewhere [2]. In brief, there were three parallel cohorts based on the product of the index dispensation aiming to recruit 10,000 patients with a celecoxib dispensation, 10,000 patients with a rofecoxib dispensation and 20,000 patients with a tNSAID dispensation. All NSAIDs with the M01A code in the World Health Organization Anatomical Therapeutic Chemical (ATC) classification were considered. Glucosamine was excluded although it belongs to the M01A group owing to its different pharmacological properties. Regarding aspirin in combination with corticosteroids (ATC code M01BA03), as it is not marketed in France it was not included in the analysis. Low-dose NSAIDs (at analgesic doses) used only for analgesia or antipyrexia as well as all forms of aspirin were excluded from the selection process, but not from the data collected. Only orally administered NSAIDs were included. Regarding previous medical history, physician-declared data were used, and previous gastrointestinal history was defined as: dyspepsia, gastro-oesophageal reflux disease (GERD), oesophageal ulcer, gastro-duodenal ulcer, perforated ulcer, or upper gastrointestinal bleeding; previous cardiovascular history was defined as: arterial hypertension, myocardial infarction, unstable angina, angina pectoris, left ventricular insufficiency, lower limb arteritis, ischemic or haemorrhagic stroke, or diabetes mellitus, hypercholesterolaemia. 


\section{Study design and study population:}

The present study population, a part of the CADEUS study, consisted of patients aged $\geq 65$ years with diagnosed OA, having filed a reimbursement claim for a tNSAID (index drug, ATC code: M01A) during the study period (01/08/2003 - 31/07/2004) and at least one tNSAID (index or other tNSAID) in the six preceding months $(n=1,851)$. Such patients were considered to have been exposed to a tNSAID.

\section{Data Analysis:}

According to the modified Beers list and the Afssaps recommendations, the dispensation of indomethacin; at least 1 dispensation of two different tNSAIDs within 1 month; at least 1 dispensation of tNSAIDs either with platelet aggregation inhibitors (ATC code B01AC), vitamin $\mathrm{K}$ antagonists (VKA) (ATC code B01AA) or aspirin $\geq 325 \mathrm{mg} /$ day (ATC code M01BA03) within 1 month were considered as inappropriate tNSAID use. To better characterize tNSAIDs inappropriate users, drug use in the previous 6 months was also considered (acid suppressing agents [proton pump inhibitors ATC code A02BC], cardiovascular agents). In France, prescribed NSAIDs are delivered in non-divisible packets containing, for the most part, 30 delivery units. Therefore, long-term use of high-dose (antiinflammatory) tNSAIDs was defined as having at least 5 tNSAID dispensations over 6 months with a gap of less than 45 days between each of these, and when a gap was greater than 45 days, medicine availability $>50 \%$ (i.e. DDD delivered/theoretical DDD for the gap). DDDs of the tNSAIDs were those listed in the ATC/DDD index 2007.

Statistical analyses were descriptive and were performed using the SAS statistical programme (SAS Institute, version 9.1, North Carolina, USA).

Regulatory aspects:

The protocol was submitted to and validated by the French National Data-protection Commission in charge of authorising data-processing (CNIL: Commission Nationale de 
l'Informatique et des Libertés) and the French National Council of Physicians (CNOM: Conseil National de l'Ordre des Médecins). All participating subjects gave written informed consent. For purely observational studies performed in France, approval from an ethics committee is not required and the CADEUS study was therefore not submitted for approval. 


\section{Results}

Within the CADEUS tNSAID cohort, 1,851 OA patients were identified according to the inclusion criteria and constituted our study population (28.4\% male; Table 1$)$. In the study population, the five most frequently reimbursed tNSAIDs over the study period were piroxicam $(25.0 \%)$, diclofenac $(24.0 \%)$, ibuprofen $(18.0 \%)$, ketoprofen $(18.0 \%)$, and naproxen $(10.0 \%)$. The maximum number of dispensations over the one-year study period was $7(n=2)$, and $22.6 \%$ of the study population were long-term users of tNSAIDs.

Prevalence of patients with at least one criterion defining inappropriate use was $43.0 \%$. Considering each criterion, indomethacin dispensation was found in $1.5 \%(\mathrm{n}=28)$ of the study population. Eleven of these patients were dispensed indomethacin only once. Within one month, $279(15.0 \%)$ were dispensed two different tNSAIDs, 85 (4.6\%) aspirin and a tNSAID, and $275(15.0 \%)$ a platelet aggregation inhibitor and a tNSAID. Across these groups, age ranged from 73.2 to 76.2 years, presence of previous cardiovascular history ranged from 60.9 to $85.8 \%$, gastrointestinal history from 29.0 to $39.3 \%$. For those with indomethacin dispensed, $39.3 \%$ did not use gastroprotective agents in the previous 6 months, neither did $41.2 \%$ of those dispensed two different tNSAIDs, $54.1 \%$ of those dispensed aspirin and tNSAIDs, or $52.0 \%$ of those dispensed a platelet aggregation inhibitor and tNSAIDs in 1 month (Table 2).

Among those dispensed two different tNSAIDs at least once within one month $(n=279)$ the most frequent combinations were ketoprofen with piroxicam (11.0\%), diclofenac with piroxicam $(10.0 \%)$, diclofenac with ibuprofen $(8.0 \%)$, and diclofenac with ketoprofen $(7.5 \%)$ (Table 3). Among those dispensed tNSAIDs with a platelet aggregation inhibitor within one month $(n=275)$, the most frequent tNSAIDs were diclofenac $(27.0 \%)$, piroxicam $(23.0 \%)$, ibuprofen (19.0\%), and ketoprofen (15.0\%) (Table 4). Of the four patients dispensed tNSAIDs with VKAs, the tNSAIDs were diclofenac $(0.05 \%)$, indomethacin $(0.05 \%)$, ketoprofen $(0.10 \%)$ and piroxicam $(0.05 \%)$. Among those dispensed tNSAIDs with low-dose 
aspirin, the most frequent tNSAIDs were piroxicam (31\%), diclofenac (22\%) or ibuprofen (19\%) (Table 5). 


\section{Discussion}

The present study describes inappropriate prescription of tNSAIDs in elderly OA patients that is variable according to the criteria considered. Beers criteria recommend that use of either indomethacin or ketorolac is inappropriate in the elderly. Only $1.5 \%$ of our study population was prescribed indomethacin. None was prescribed ketorolac, which is no longer on the French market. Only four subjects were co-dispensed a tNSAID with a VKA. On the other hand, $4.6 \%$ of the elderly patients concomitantly used low-dose aspirin, and $15 \%$ each were prescribed two different tNSAIDs at least once within a month, or co-prescribed APA.

Since October 2005, three NSAIDs have been given particular attention in Europe and in France: ketoprofen and piroxicam [12]. For piroxicam, an arbitrage procedure was triggered due to its serious digestive and cutaneous side effects. For ketoprofen, the risk/benefit ratio still stays positive but the daily dosage should not exceed $200 \mathrm{mg}$. In our study cohort, $25 \%$ and $20 \%$ of the study population were prescribed piroxicam and ketoprofen at least once, respectively.

Traditional NSAIDs should be used at the minimum effective dose and for the shortest possible duration [11]. Since the 2003 Beers criteria did not define high dose and long-term NSAIDs utilization, this was defined for the current investigate. All tNSAIDs were included in the analysis of high dose and long-term use even though Beers criteria specifically finger naproxen, oxaprozin, piroxicam, whereas Afsappss does not specify whether any drugs are at specific risk. Even though $\mathrm{OA}$ is a chronic painful disease, only $22.6 \%$ of our study population were users of tNSAIDs with high dose and long duration, which is consistent with known patterns of use of NSAIDs in France [1, 13, 14].

Prescription of two or more NSAIDs is not recommended especially in the elderly due to increased risk of side effects. In our study population, prevalence of dispensation of two NSAIDs was $15 \%$. However, as dispensed drugs may not be taken by the patient, this may not 
reflect the true prevalence of concomitant intake. It might also indicate prescriber modulation of prescription according to symptoms, with use of longer-duration of riskier drugs (e.g., piroxicam for flares or exacerbations of the disease), and shorter-acting NSAIDs such as ibuprofen, ketoprofen or diclofenac for less severe episodes. The main problem of multiple NSAIDs use is their increased risk of GI toxicity related to the overall NSAID burden. In these cases, use of gastroprotective agents might alleviate the risk, and it is interesting to see that it is in this population and in patients prescribed indomethacin that there was the lower rate of non-users of gastroprotective agents (Table 2).

Many studies have established the increased gastrointestinal toxicity of tNSAIDs and therefore the use of gastroprotective agents for patients with risk factors for gastrointestinal complications are highly recommended [15]; i.e. age over 65 years, history of a peptic ulcer and concomitant use of anticoagulants drugs, acetylsalicylic acid. In our study population, the prevalence of gastroprotective agent users among chronic NSAIDs users (chronic and continuous) ranged between 36 to $60 \%$ for each criterion.

One of the limitations of the present study could be that only patients registered in the French general healthcare insurance system were included in the whole CADEUS cohort, however, this system does cover $85 \%$ of the French population. Beers Criteria have become the most widely cited criteria for inappropriate prescription in the elderly, nevertheless, some deficiencies are known $[16,17]$. As the last version of these was published in 2003, they need updating, in particular, with respect to non-selective NSAIDs versus COX-2 selective NSAIDs, and the concomitant use of gastroprotective drugs, which alleviate some of the risks related to NSAIDs in the elderly, though other risks are not modified (e.g., renal failure, or cardiovascular events). These would need to be carefully assessed to rejuvenate these criteria and avoid leaving patients who need NSAIDs deprived of them. 
The elderly are at significant risk for increased adverse drug reactions for several reasons and therefore physicians should be more cautious when prescribing tNSAIDs to elderly patients especially who have risk factors. Appropriate conduct would be avoiding these drugs when possible (whilst acknowledging that substitutes such as paracetamol or opiates are not wholly devoid of problems too), or using measures to counter the risk such as GPA, and especially carefully monitoring these patients and their therapeutic options.

\section{Conclusion}

The more common inappropriate NSAIDs prescription was co-prescription of different NSAIDs within a month, and co-prescription with platelet aggregation inhibitor. The real-life consequences of these needs to be ascertained. 


\section{Competing interests}

The authors declare that they have no conflict of interest.

\section{Authors' contributions}

SEG, AFR, CDP, RL, MR conceived the study and participated in its design. SEG, RL carried out acquisition, statistical analysis, and interpretation of data. SEG drafted the manuscript. SEG, CDP, RL, PB, BB, MR, NM and AFR participated in the critical revision of the manuscript for important intellectual content. All authors have given final approval of the version to be published. 


\section{Acknowledgement:}

We thank Philip Robinson for help in manuscript preparation, who is an employee of the Département de Pharmacologie, Université de Bordeaux , Bordeaux, France.

We would like to thank all those who participated in the CADEUS study, and the patients and the prescribers who provided the data.

The CADEUS study was supported by Pfizer Inc. and Merck and Co. The funding source had no involvement in study design; collection, analysis or interpretation of data; in the writing of the report; or in the decision to submit the paper for publication. The present analysis of CADEUS data was entirely funded by the Départment de Pharmacologie, INSERM U657, Université de Bordeaux . 


\section{References}

1 Moore N, Diris H, Martin K, Viale R, Fourrier A, Moride Y, Begaud B (2004) NSAID use profiles derived from reimbursement data in France. Therapie 59 (5): 541-546

2 Depont F, Fourrier A, Merliere Y, Droz C, Amouretti M, Begaud B, Benichou J, Moride Y, Blin P, Moore N (2007) The CADEUS study: methods and logistics. Pharmacoepidemiol Drug Saf 16 (5): 571-580

3 Depont F, Fourrier A, Merliere Y, Droz C, Amouretti M, Begaud B, Benichou J, Moride Y, Velo GP, Sturkenboom M, Blin P, Moore N (2007) Channelling of COX-2 inhibitors to patients at higher gastrointestinal risk but not at lower cardiovascular risk: the Cox 2 inhibitors and tNSAIDs description of users (CADEUS) study. Pharmacoepidemiol Drug Saf 16 (8): 891-900

4 Vane JR (1971) Inhibition of prostaglandin synthesis as a mechanism of action for aspirin-like drugs. Nat New Biol 231 (25): 232-235

5 Silverstein FE, Faich G, Goldstein JL, Simon LS, Pincus T, Whelton A, Makuch R, Eisen G, Agrawal NM, Stenson WF, Burr AM, Zhao WW, Kent JD, Lefkowith JB, Verburg KM, Geis GS (2000) Gastrointestinal toxicity with celecoxib vs nonsteroidal anti-inflammatory drugs for osteoarthritis and rheumatoid arthritis: the CLASS study: A randomized controlled trial. Celecoxib Long-term Arthritis Safety Study. JAMA 284 (10): 1247-1255

6 Bombardier C, Laine L, Reicin A, Shapiro D, Burgos-Vargas R, Davis B, Day R, Ferraz MB, Hawkey CJ, Hochberg MC, Kvien TK, Schnitzer TJ (2000) Comparison of upper gastrointestinal toxicity of rofecoxib and naproxen in patients with rheumatoid arthritis. VIGOR Study Group. N Engl J Med 343 (21): 1520-1528, 1522 p following 1528 
7 Hermann M (2009) Cardiovascular risk associated with nonsteroidal antiinflammatory drugs. Curr Rheumatol Rep 11 (1): 31-35 Beers MH (1997) Explicit criteria for determining potentially inappropriate medication use by the elderly. An update. Arch Intern Med 157 (14): 1531-1536

9 Beers MH, Ouslander JG, Rollingher I, Reuben DB, Brooks J, Beck JC (1991) Explicit criteria for determining inappropriate medication use in nursing home residents. UCLA Division of Geriatric Medicine. Arch Intern Med 151 (9): 1825-1832

10 Fick DM, Cooper JW, Wade WE, Waller JL, Maclean JR, Beers MH (2003) Updating the Beers criteria for potentially inappropriate medication use in older adults: results of a US consensus panel of experts. Arch Intern Med 163 (22): 2716-2724

11 AFSSAPS (2006) Rappel des règles de bon usage des AINS. AFSSAPS

12 AFSSAPS (2006) Evaluation des nouvelles données de tolérance cardiovasculaire : Le rapport bénéfice/risque des AINS reste favorable mais les précautions d'emploi doivent être renforcées. In: AFSSAPS-Communiqué de presseed.

13 Moore N (2003) Place of OTC analgesics and NSAIDs in osteoarthritis. Inflammopharmacology 11 (4): 355-362

14 Moore N, Verschuren X, Montout C, Callens J, Kong SX, Begaud B (2000) Excess costs related to non-steroidal anti-inflammatory drug utilization in general practice. Therapie 55 (1): 133-136

15 Becker JC, Domschke W, Pohle T (2004) Current approaches to prevent NSAIDinduced gastropathy--COX selectivity and beyond. Br J Clin Pharmacol 58 (6): 587600

16 Gallagher PF, Barry PJ, Ryan C, Hartigan I, O'Mahony D (2008) Inappropriate prescribing in an acutely ill population of elderly patients as determined by Beers' Criteria. Age Ageing 37 (1): 96-101 
17 O'Mahony D, Gallagher PF (2008) Inappropriate prescribing in the older population: need for new criteria. Age Ageing 37 (2): 138-141 
Table 1. General characteristics of OA patients.

\begin{tabular}{ll}
\hline Characteristics & All patients $(\mathrm{n}=1,851)$ \\
\hline Age, mean (SD) & $76.3(6.4)$ \\
Male & $525(28.364)$ \\
Current drug use & \\
- Proton pump inhibitors & $679(36.82)$ \\
- Cardiovascular medications & $984(53.126)$ \\
History of & \\
- Cardiovascular disorders & $1,211(65.576)$ \\
- Cerebrovascular ischemia & $38(2.346)$ \\
- Hypercholesterolemia & $523(31.13)$ \\
- Past history of digestive events & $558(30.152)$ \\
\hline
\end{tabular}

Unless otherwise indicated, data are shown as numbers (\%). 
Table 2. General characteristics of OA patients stratified according to each Beers criterion.

\begin{tabular}{|c|c|c|c|c|}
\hline & & \multicolumn{3}{|c|}{ Within 1 month } \\
\hline & $\begin{array}{l}\text { Indomethacin at } \\
\text { least once } \\
(n=28)\end{array}$ & $\begin{array}{l}\text { Two } \\
\text { different } \\
\text { tNSAID } \\
(n=279)\end{array}$ & $\begin{array}{l}\text { Aspirin }+ \\
\geq 1 \mathrm{tNSAID} \\
(\mathrm{n}=85)\end{array}$ & $\begin{array}{l}\text { Platelet } \\
\text { aggregation } \\
\text { inhibitors }+\geq 1 \\
\text { tNSAID } \\
(n=275)\end{array}$ \\
\hline Age, years (mean) & $75.1(6.2)$ & $73.2(5.9)$ & $76.2(7.3)$ & $75.8(6.5)$ \\
\hline Male & $8(28.6)$ & $73(26.2)$ & $27(31.8)$ & $106(38.5)$ \\
\hline \multicolumn{5}{|l|}{ Previous medical history } \\
\hline - Cardiovascular disorders & $20(71.4)$ & $170(60.9)$ & $56(65.9)$ & $236(85.8)$ \\
\hline - Gastrointestinal events & $11(39.3)$ & $84(30.1)$ & $25(29.0)$ & $95(34.5)$ \\
\hline \multicolumn{5}{|l|}{ PPI use in previous 6 months } \\
\hline [dispensations, n] & & & & \\
\hline - Non-users & $11(39.3)$ & $115(41.2)$ & $46(54.1)$ & $143(52.0)$ \\
\hline - Occasional users [1-3] & $1(3.6)$ & $46(16.5)$ & $8(9.4)$ & $25(9.1)$ \\
\hline - Intermittent users [4-9] & $6(21.4)$ & $46(16.5)$ & $16(18.8)$ & $28(10.2)$ \\
\hline - Continuous users $[\geq 10]$ & $10(35.7)$ & $72(25.8)$ & $15(17.6)$ & $79(28.7)$ \\
\hline
\end{tabular}


Table 3. The most frequent $(n \geq 5)$ combinations of two different $t$ NSAIDs dispensed at least once within the same month.

$\mathrm{n}=279$

\begin{tabular}{lc}
\hline Two different tNSAIDs, $\mathrm{n}(\%)$ & $30(10.7)$ \\
ketoprofen + piroxicam & $29(10.4)$ \\
diclofenac + piroxicam & $22(7.9)$ \\
diclofenac + ibuprofen & $21(7.5)$ \\
diclofenac + ketoprofen & $14(5.0)$ \\
diclofenac + naproxen & $14(5.0)$ \\
ketoprofen + tenoxicam & $11(3.9)$ \\
ibuprofen + piroxicam & $11(3.9)$ \\
ibuprofen + ketoprofen & $10(3.6)$ \\
ketoprofen + naproxen & $10(3.6)$ \\
ketoprofen + nimesulide & $8(2.8)$ \\
naproxen + piroxicam & $8(2.8)$ \\
meloxicam + piroxicam & $7(2.5)$ \\
piroxicam + tenoxicam & $5(1.8)$ \\
ibuprofen + meloxicam & \\
\hline
\end{tabular}


Table 4. tNSAIDs most frequently $(n \geq 5)$ dispensed in combination (within the same month) with platelet aggregation inhibitors (ATC code B01AC).

tNSAIDs in combination with platelet $\quad \mathrm{n}(\%)$

aggregation inhibitors, $\mathrm{n}=275$

Diclofenac

$75(27.3)$

Piroxicam

$63(22.9)$

Ibuprofen

$52(18.9)$

Ketoprofen

$40(14.5)$

Flurbiprofen

$23(30.7)$

Naproxen

$20(26.7)$

Nimesulide

$19(6.9)$

Meloxicam

$15(20.0)$

Diclofenac combinations

$14(5.1)$

Tenoxicam

$10(13.3)$ 
Table 5. tNSAIDs most frequently ( $\mathrm{n} \geq 5$ ) dispensed in combination (within the same month) with low-dose aspirin (ATC code M01BA03).

tNSAIDs in combination with low-dose aspirin, $\quad \mathrm{n}(\%)$

$\mathrm{n}=85$

Piroxicam

$26(30.5)$

Diclofenac

$19(22.3)$

Ibuprofen

$16(18.8)$

Naproxen

$7(8.2)$

Diclofenac combinations

$5(5.8)$

Ketoprofen

$5(5.8)$

Etodolac

$2(2.3)$ 